\title{
A kind of extension of the famous Young inequality
}

\author{
Luo Xianqiang ${ }^{1,2^{*}}$ and Xiong Zhiping ${ }^{2}$
}

\section{*Correspondence:}

luoxq1978@126.com

'Department of Mathematics,

Shanghai University, Shanghai,

200444, China

${ }^{2}$ Mathematics College, Wuyi

University, Guangdong, 529020,

China

\begin{abstract}
Young inequality, extended in (Geometry of Orlicz Spaces, 1986; Geometry of Orlicz Spaces, 1996), has extensive use and great effort in mathematical analysis. By the kind of extended Young inequality, we can get the famous Holder inequality and the Minkowski inequality. But until now, we have not found its strict proof of analysis. In (Geometry of Orlicz Spaces, 1986; Geometry of Orlicz Spaces, 1996), only the probable pattern description was found. In this paper, we will get the strict proof of analysis of a kind of extension of Young inequality with the approximation method.
\end{abstract}

MSC: 46B20; 46B02; 46A22

Keywords: Young inequality; $N$-function; strictly convex function

\section{Introduction}

The original Young inequality [1] has been proposed in an integral form by Young in 1912. Suppose that $f(x)$ is a strictly increasing and continuous function defined in $[0, c], f^{-1}(x)$ is the inverse function of $f(x), f(0)=0, a \in[0, c], b \in[f(0), f(c)]$. Then

$$
\int_{0}^{a} f(x) d x+\int_{0}^{b} f^{-1}(x) d x \geq a b
$$

where the equality holds if and only if $b=f(a)$.

Young inequality has an extensive use and a great effort in mathematical analysis. Now Young inequality was extended as follows.

Let $M(u)$ and $N(v)$ be complementary $N$-function with each other (see Definition 2.1 and Definition 2.2), then the kind of Young inequality $u v \leq M(u)+N(v)$ holds, and the equality holds if and only if $u=q(|v|) \operatorname{sign} v$ or $v=p(|u|) \operatorname{sign} u$ for all $u, v \in(-\infty,+\infty)$.

By the kind of Young inequality, we can get the famous Holder inequality and the Minkowski inequality (see references [2] and [3]). But until now, we have not found its strict proof of analysis. In references [2] and [3], only the probable pattern description was found. Some other decisions can be found in [4-8].

In this paper, we will get its strict proof of analysis with the approximation method.

\section{Preliminaries}

Definition 2.1 [2] The mapping $M:(-\infty,+\infty) \rightarrow(-\infty,+\infty)$ is called an $N$-function if it has the following properties:

(i) $M(u)$ is even, continuous, convex and $M(0)=0$.

C) 2013 Xianqiang and Zhiping; licensee Springer. This is an Open Access article distributed under the terms of the Creative Commons Attribution License (http://creativecommons.org/licenses/by/2.0), which permits unrestricted use, distribution, and reproduction in any medium, provided the original work is properly cited. 
(ii) $M(u)>0$ for all $u \neq 0$.

(iii) $\lim _{u \rightarrow 0} \frac{M(u)}{u}=0$ and $\lim _{u \rightarrow \infty} \frac{M(u)}{u}=\infty$.

Lemma 2.1 [2] $M(u)$ is an $N$-function if and only if there exists $p(u):[0,+\infty) \rightarrow[0,+\infty)$ with the following properties:

(i) $p(u)$ is right-continuous and nondecreasing;

(ii) $p(u)>0$ whenever $u \neq 0$;

(iii) $p(0)=0$ and $p(\infty)=\infty, M(u)=\int_{0}^{|u|} p(t) d t$.

Record 2.1 [2] $p(u)$ is the right-derivative of $N$-function $M(u)$.

Lemma 2.2 Let $p_{-}(u)$ be the left-derivative of $N$-function $M(u)$, then $p_{-}(u)=\lim _{h \rightarrow 0^{+}} p(u-$ $h)$, and $\int_{0}^{|u|} p_{-}(t) d t=M(u)$.

Proof From the proof process of Theorem 1.4 in reference [2], we know $p_{-}(u)$ is left continuous, and for all $0<u<v, p(u) \leq p_{-}(v) \leq p(v)$.

Hence, for $h>0$, we have $p(v-h) \leq p_{-}(v)$.

Therefore,

$$
\lim _{h \rightarrow 0^{+}} p(v-h) \leq p_{-}(v)
$$

On the other hand, since $p_{-}(v) \leq p(v)$ and $p_{-}(v)$ is left continuous, we get

$$
p_{-}(v)=\lim _{h \rightarrow 0^{+}} p_{-}(v-h) \leq \lim _{h \rightarrow 0^{+}} p(v-h) .
$$

Therefore, we have

$$
p_{-}(v)=\lim _{h \rightarrow 0^{+}} p(v-h) \text {. }
$$

Since for all $h>0, p(v-h) \leq p_{-}(v) \leq p(v)$, then we have

$$
\int_{0}^{|u|} p(t-h) d t \leq \int_{0}^{|u|} p_{-}(t) d t \leq \int_{0}^{|u|} p(t) d t=M(u) .
$$

That is,

$$
M(|u|-h)-M(-h) \leq \int_{0}^{|u|} p_{-}(t) d t \leq M(u) .
$$

Let $h \rightarrow 0$, by the property (i) of $M(u)$ in Definition 2.1, we have

$$
\int_{0}^{|u|} p_{-}(t) d t=M(u)
$$

Definition 2.2 [2] Suppose that $M(u)$ is an $N$-function. Let $p(t)$ be the right derivative of $M(u)$. Let $q(s)=\sup _{p(t) \leq s} t=\inf _{p(t)>s} t$, called the right-inverse function of $p(t)$. By Theorem 1.5 in reference [2], we know that $q(s)$ also satisfies the three properties of Lemma 2.1, and $N(v)=\int_{0}^{|v|} q(s) d s$ is called the complementary $N$-function of $M(u)$. It is obvious, the left derivative $q_{-}(s)$ of $N(v)$ satisfies $q_{-}(s)=\sup _{p(t)<s} t=\inf _{p(t) \geq s} t$. 
Lemma 2.3 [2] $q(p(t)) \geq t, p(q(s)) \geq s ; q(p(t)-\varepsilon) \leq t, p(q(s)-\varepsilon) \leq s$.

Lemma 2.4 [2] $M(u)$ is strictly convex if and only if $p(t)$ is strictly increasing, that is, $q(s)$ is continuous.

Lemma 2.5 [2] For any $N$-function $M(u)$ and $\varepsilon>0$, there exists a strictly convex $N$ function $M_{1}(u)$, such that

$$
(1-\varepsilon) p(t) \leq p_{1}(t) \leq(1+\varepsilon) p(t), \quad(1-\varepsilon) M(u) \leq M_{1}(u) \leq(1+\varepsilon) M(u),
$$

where $p(t)$ and $p_{1}(t)$ are the right derivatives of $M(u)$ and $M_{1}(u)$, respectively.

Record 2.2 Lemma 2.5 is Theorem 1.10 in reference [2], but it reverses the old conclusion ' $M(u) \leq M_{1}(u) \leq(1+\varepsilon) M(u)$, for the new conclusion ' $(1-\varepsilon) M(u) \leq M_{1}(u) \leq(1+\varepsilon) M(u)$.' From the construction process of $p_{1}(t)$, in the proof in reference [3], we know if $p(t)$ is continuous, then $p_{1}(t)$ is also continuous.

Lemma 2.6 Suppose that $u \geq 0$ and $v \geq 0$, then $u=q(v)$ or $v=p(u)$ if and only if $u \in$ $\left[q_{-}(v), q(v)\right]$. By the symmetry, we get another necessary and sufficient condition, that is, $v \in\left[p_{-}(u), p(u)\right]$.

\section{Proof Sufficiency.}

Suppose that $u \in\left[q_{-}(v), q(v)\right]$.

(i) If $q_{-}(v)=q(v)$, it is clear that $u=q_{-}(v)=q(v)$.

(ii) If $q_{-}(v) \neq q(v)$, then $q_{-}(v)<q(v)$. If $u=q(v)$, then the conclusion holds.

If $q_{-}(v) \leq u<q(v)$, we need only to prove that $p(u)=v$.

From Definition 2.2, we have

$$
q_{-}(v)=\sup _{p(t)<v} t=\inf _{p(t) \geq v} t
$$

Since $q_{-}(v) \leq u \Rightarrow \sup _{p(t)<v} t \leq u$, then for any $\frac{1}{n}$, we get

$$
p\left(u+\frac{1}{n}\right) \geq v
$$

Let $n \rightarrow \infty$, since $p(t)$ is right continuous, then we have

$$
p(u) \geq v
$$

On the other hand, from $u<q(v)=\sup _{p(t) \leq v} t=\inf _{p(t) \geq v} t$, we get

$$
p(u) \leq v .
$$

So, we have

$$
p(u)=v
$$


Necessity.

If $u=q(v)$, it is clearly established.

If $p(u)=v$, then from

$$
q_{-}(v)=\inf _{p(t) \geq v} t \leq u
$$

and

$$
q(v)=\sup _{p(t) \leq v} t \geq u
$$

We have

$$
u \in\left[q_{-}(v), q(v)\right] .
$$

The next two lemmas are about the change of variable of integral and distribute integral.

Lemma 2.7 [9] Suppose thatf $(x)$ and $g(x)$ are defined on the interval $[a, b]$, and the Stieltjes integral of $f(x)$ about $g(x)$ exists. Suppose that $x(t)$ is a strictly increasing and continuous function on the interval $[\alpha, \beta]$, and $x(\alpha)=a$ and $x(\beta)=b$, then

$$
\int_{\alpha}^{\beta} f(x(t)) d g(x(t))=\int_{a}^{b} f(x) d g(x) .
$$

Lemma 2.8 [9] Suppose that $f(x)$ and $g(x)$ are defined on the interval $[a, b]$, and the Stieltjes integral of $f(x)$ about $g(x)$ exists, then

$$
\int_{a}^{b} f(x) d g(x)+\int_{a}^{b} g(x) d f(x)=f(b) g(b)-f(a) g(a) .
$$

\section{Main result}

Theorem 3.1 Suppose that $M(u)$ is an $N$-function, and $N(v)$ is the complementary $N$ function of $M(u)$, then Young inequality $u v \leq M(u)+N(v)$ holds, and $u v=M(u)+N(v)$ holds if and only if $u=q(|v|) \operatorname{sign} v$ or $v=p(|u|) \operatorname{sign} u$.

Proof Suppose that $u \geq 0$ and $v \geq 0$.

Firstly, we will prove the necessity of the equality.

Suppose that there exist $u_{0} \geq 0$ and $v_{0} \geq 0$ satisfying

$$
M\left(u_{0}\right)+N\left(v_{0}\right)=u_{0} v_{0} .
$$

Let

$$
F(u, v)=M(u)+N(v)-u v .
$$

From Young inequality, we have learned that for all $u$ and $v, F(u, v) \geq 0$.

From $M\left(u_{0}\right)+N\left(v_{0}\right)=u_{0} v_{0}$, we have $F\left(u, v_{0}\right)=M(u)+N\left(v_{0}\right)-u v_{0}$ and we can get the minimum 0 in $u_{0}$. 
If $u_{0}=0$, from $M\left(u_{0}\right)+N\left(v_{0}\right)=u_{0} v_{0}$, we get that $v_{0}=0$, then $u_{0}=q\left(v_{0}\right)=0$ or $v_{0}=$ $p\left(u_{0}\right)=0$, that is, the necessity of the equality holds.

If $u_{0} \neq 0$, then $F\left(u_{0}, v_{0}\right)$ is the minimum of the $F\left(u, v_{0}\right)$ on the interval $(0,+\infty)$.

Therefore, the left derivative of $F\left(u, v_{0}\right)$ is less than or equal to zero on the point $u_{0}$, and the right derivative of $F\left(u, v_{0}\right)$ is more than or equal to zero on the point $u_{0}$.

That is,

$$
p_{-}\left(u_{0}\right)-v_{0} \leq 0, p\left(u_{0}\right)-v_{0} \geq 0 \text {. }
$$

Then

$$
v_{0} \in\left[p_{-}\left(u_{0}\right), p\left(u_{0}\right)\right]
$$

From Lemma 2.6, we get $u_{0}=q\left(v_{0}\right)$ or $v_{0}=p\left(u_{0}\right)$.

That is, the necessity of the equality holds.

Secondly, we will get the proof of the Young inequality and the sufficiency of the equality in three steps.

Step I. Suppose that $M(u)$ and $N(v)$ are all strictly convex. From Lemma 2.4, the right derivative $p(t)$ and $q(s)$ are all strictly increasing, continuous, and are the right inversefunction of each other. From the reference [9], we have that the Stieltjes integral $\int_{0}^{q(v)} t d p(t)$ exists.

From Lemma 2.7 and Lemma 2.8, we have

$$
\begin{aligned}
M(u)+N(v) & \\
= & \int_{0}^{u} p(t) d t+\int_{0}^{v} q(s) d s \\
= & \int_{0}^{u} p(t) d t+\int_{0}^{q(v)} t d p(t) \\
= & \int_{0}^{u} p(t) d t+v q(v)-\int_{0}^{q(v)} p(t) d t \\
= & v q(v)+\int_{q(v)}^{u} p(t) d t .
\end{aligned}
$$

(i) If $u>q(v)$, then $p(u)>v$.

Hence, by expression (1), we have

$$
\begin{aligned}
& M(u)+N(v) \\
& =\int_{q(v)}^{u} p(t) d t+v q(v) \\
& =\int_{q(v)}^{u} p(t) d t+u v-v(u-q(v)) \\
& \geq p(q(v))(u-q(v))+u v-v(u-q(v)) \\
& =u v .
\end{aligned}
$$

(ii) If $u<q(v)$, then $p(u) \leq v$. 
Hence, by expression (1), we have

$$
\begin{aligned}
& M(u)+N(v) \\
& \quad=v q(v)-\int_{u}^{q(v)} p(t) d t \\
& \quad=u v+v(q(v)-u)-\int_{u}^{q(v)} p(t) d t \\
& \quad \geq u v+v(q(v)-u)-p(q(v))(q(v)-u) \\
& \quad=u v .
\end{aligned}
$$

(iii) If $u=q(v)$, then $v=p(u)$.

From expression (1), we have $u v=M(u)+N(v)$.

That is, the sufficiency of the equality holds.

Step II. Suppose that $M(u)$ is strictly convex, then from Lemma 2.4 , the right derivative $p(t)$ is strictly increasing, and the right-inverse function $q(s)$ is continuous and nondecreasing.

From Lemma 2.5 and Record 2.2, $\forall 0<\varepsilon<\frac{1}{2}$, we can construct a function strictly increasing and continuous $q_{1}(s)$ such that

$$
(1-\varepsilon) q(s) \leq q_{1}(s) \leq(1+\varepsilon) q(s) .
$$

Hence,

$$
\frac{1}{1+\varepsilon} q_{1}(s) \leq q(s) \leq \frac{1}{1-\varepsilon} q_{1}(s)
$$

Let $p_{1}(t)$ be the right-inverse function of $q_{1}(s)$, then $p_{1}(t)$ is strictly increasing and continuous.

In the following, we will get the relation of $p_{1}(t)$ and $p(t)$.

In expression (2), let $s=p_{1}(t)$, we have

$$
\frac{1}{1+\varepsilon} q_{1}\left(p_{1}(t)\right) \leq q\left(p_{1}(t)\right) \leq \frac{1}{1-\varepsilon} q_{1}\left(p_{1}(t)\right)
$$

That is,

$$
\frac{t}{1+\varepsilon} \leq q\left(p_{1}(t)\right) \leq \frac{t}{1-\varepsilon} .
$$

From Lemma 2.3 and expression (3), we get

$$
q\left(p\left(\frac{t}{1-2 \varepsilon}\right)\right) \geq \frac{t}{1-2 \varepsilon}>\frac{t}{1-\varepsilon} \geq q\left(p_{1}(t)\right) .
$$

Since $q(s)$ is nondecreasing, by expression (4), we get

$$
p\left(\frac{t}{1-2 \varepsilon}\right)>p_{1}(t)
$$


From the result in Step I, we get

$$
u v \leq M_{1}(u)+N_{1}(v)=\int_{0}^{u} p_{1}(t) d t+\int_{0}^{v} q_{1}(s) d s
$$

Therefore,

$$
\begin{aligned}
M(u)+N(v) & \\
& =\int_{0}^{u} p(t) d t+\int_{0}^{v} q(s) d s \\
& \geq \int_{0}^{u} p(t) d t+\int_{0}^{v} \frac{1}{1+\varepsilon} q_{1}(s) d s \quad(\mathrm{by}(2)) \\
\geq & \int_{0}^{u} p(t) d t+\frac{1}{1+\varepsilon}\left(u v-\int_{0}^{u} p_{1}(t) d t\right) \\
> & \frac{1}{1+\varepsilon} u v+\int_{0}^{u} p(t) d t-\frac{1}{1+\varepsilon} \int_{0}^{u} p\left(\frac{t}{1-2 \varepsilon}\right) d t \quad \text { (by (5)) } \\
= & \frac{1}{1+\varepsilon} u v+\int_{0}^{u} p(t) d t-\frac{1-2 \varepsilon}{1+\varepsilon} \int_{0}^{\frac{u}{1-2 \varepsilon}} p(t) d t .
\end{aligned}
$$

Let $\varepsilon \rightarrow 0$, we have

$$
M(u)+N(v) \geq u v .
$$

In the following, we will prove the sufficiency of the equality.

If $v=p(u)$, from Lemma 2.3 and expression (3), for $0<\varepsilon<\frac{1}{2}$ above, we have

$$
q\left(p\left(\frac{t}{1+2 \varepsilon}\right)-\varepsilon\right) \leq \frac{t}{1+2 \varepsilon}<\frac{t}{1+\varepsilon} \leq q\left(p_{1}(t)\right) \quad \Rightarrow \quad p\left(\frac{t}{1+2 \varepsilon}\right)-\varepsilon<p_{1}(t)
$$

In expression (6), let $\varepsilon \rightarrow 0$, by Lemma 2.2 , we get

$$
p_{-}(t) \leq \lim _{\varepsilon \rightarrow 0} p_{1}(t)
$$

On the other hand, in expression (5), let $\varepsilon \rightarrow 0$, we get

$$
\lim _{\varepsilon \rightarrow 0} p_{1}(t) \leq p(t)
$$

Therefore,

$$
p_{-}(t) \leq \lim _{\varepsilon \rightarrow 0} p_{1}(t) \leq p(t)
$$

By Lemma 2.2, we get

$$
\int_{0}^{u} p_{-}(t) d t=M(u)=\int_{0}^{u} \lim _{\varepsilon \rightarrow 0} p_{1}(t) d t=\int_{0}^{u} p(t) d t
$$


Now we need to prove that

$$
\int_{p_{1}(u)}^{p(u)} q(s) d s=u\left(p(u)-p_{1}(u)\right)
$$

In fact, if $s=p(u)$, from Definition 2.2, since $p(u)$ is strictly increasing, then we have $q(s)=\sup _{p(t) \leq s} t=\sup _{p(t) \leq p(u)} t=u$. If $s \in\left[p_{-}(u), p(u)\right)$, from Lemma 2.6, we get $q(s)=u$. Therefore, we have $\int_{p_{1}(u)}^{p(u)} q(s) d s=\int_{p_{1}(u)}^{p(u)} u d s=u\left(p(u)-p_{1}(u)\right)$.

By the result in Step I, we have

$$
u p_{1}(u)=M_{1}(u)+N_{1}\left(p_{1}(u)\right)=\int_{0}^{u} p_{1}(t) d t+\int_{0}^{p_{1}(u)} q_{1}(s) d s .
$$

From expressions (9) and (11), we get

$$
\begin{aligned}
M(u)+N(p(u)) & \\
= & \int_{0}^{u} p(t) d t+\int_{0}^{p(u)} q(s) d s \\
= & \int_{0}^{u} p(t) d t+\int_{0}^{p_{1}(u)} q(s) d s+\int_{p_{1}(u)}^{p(u)} q(s) d s \\
\leq & \left.\int_{0}^{u} p(t) d t+\int_{0}^{p_{1}(u)} \frac{1}{1-\varepsilon} q_{1}(s) d s+\int_{p_{1}(u)}^{p(u)} q(s) d s \quad \text { (by }(2)\right) \\
= & \int_{0}^{u} p(t) d t+\left(\frac{1}{1-\varepsilon}\right)\left(u p_{1}(u)-\int_{0}^{u} p_{1}(t) d t\right)+\int_{p_{1}(u)}^{p(u)} q(s) d s \quad \text { (by (11)) } \\
= & \int_{0}^{u} p(t) d t-\frac{1}{1-\varepsilon} \int_{0}^{u} p_{1}(t) d t+\frac{1}{1-\varepsilon} u p_{1}(u)+u\left(p(u)-p_{1}(u)\right) \quad \text { (by (10)) } \\
= & M(u)-\frac{1}{1-\varepsilon} \int_{0}^{u} p_{1}(t) d t+\left(\frac{u}{1-\varepsilon}-u\right) p_{1}(u)+u p(u) .
\end{aligned}
$$

Let $\varepsilon \rightarrow 0$, we have

$$
M(u)+N(p(u)) \leq u p(u)
$$

On the other hand, we have got the inequality $u v \leq M(u)+N(v)$.

Let $v=p(u)$, we have

$$
u p(u) \leq M(u)+N(p(u))
$$

Therefore, together with expression (12), we have

$$
M(u)+N(p(u))=u p(u) .
$$

That is, the sufficiency of the equality holds.

Step III for any $N$-function $M(u)$, suppose that its complementary $N$-function is $N(v)$, $p(t)$ is the right-inverse function of $M(u)$, and $q(s)$ is the right-inverse function of $N(v)$. 
From Lemma 2.5, for $0<\varepsilon<\frac{1}{2}$ above, we can find a strictly convex $N$-function $M_{1}(u)$ and its right-derivative $p_{1}(t)$ such that

$$
(1-\varepsilon) p(t) \leq p_{1}(t) \leq(1+\varepsilon) p(t), \quad(1-\varepsilon) M(u) \leq M_{1}(u) \leq(1+\varepsilon) M(u) .
$$

Suppose that $N_{1}(v)$ is the complementary $N$-function of $M_{1}(u), q_{1}(s)$ is the right derivative of $N_{1}(v)$.

In the following, we will get the relation of $q_{1}(t)$ and $q(t)$ for $0<\varepsilon<\frac{1}{2}$ above. In expression (13), let $t=q_{1}(s)-\varepsilon$, we have

$$
(1-\varepsilon) p\left(q_{1}(s)-\varepsilon\right) \leq p_{1}\left(q_{1}(s)-\varepsilon\right) \leq(1+\varepsilon) p\left(q_{1}(s)-\varepsilon\right) .
$$

From Lemma 2.3, we have that

$$
p_{1}\left(q_{1}(s)-\varepsilon\right) \leq s, \quad p_{1}\left(q_{1}(s)\right) \geq s .
$$

Therefore, by expressions (14) and (15), we have

$$
p\left(q_{1}(s)-\varepsilon\right) \leq \frac{s}{1-\varepsilon}, \quad p\left(q_{1}(s)\right) \geq \frac{s}{1+\varepsilon} .
$$

Then, by Lemma 2.3, together with expression (16), we have

$$
\begin{aligned}
& p\left(q_{1}(s)-\varepsilon\right) \leq \frac{s}{1-\varepsilon}<\frac{s}{1-2 \varepsilon} \leq p\left(q\left(\frac{s}{1-2 \varepsilon}\right)\right), \\
& p\left(q_{1}(s)\right) \geq \frac{s}{1+\varepsilon}>\frac{s}{1+2 \varepsilon} \geq p\left(q\left(\frac{s}{1+2 \varepsilon}\right)-\varepsilon\right) .
\end{aligned}
$$

Since $p(t)$ is nondecreasing, then by expression (17), we get

$$
q\left(\frac{s}{1+2 \varepsilon}\right)-\varepsilon<q_{1}(s)<q\left(\frac{s}{1-2 \varepsilon}\right)+\varepsilon .
$$

From the result in Step II, we get

$$
\begin{aligned}
u v & \leq M_{1}(u)+N_{1}(v) \\
& \leq(1+\varepsilon) M(u)+\int_{0}^{v} q_{1}(s) d s \\
& <(1+\varepsilon) M(u)+\int_{0}^{v}\left(q\left(\frac{s}{1-2 \varepsilon}\right)+\varepsilon\right) d s \quad \text { by (18)) } \\
& =(1+\varepsilon) M(u)+\varepsilon v+(1-2 \varepsilon) \int_{0}^{\frac{v}{1-2 \varepsilon}} q(s) d s \\
& =(1+\varepsilon) M(u)+\varepsilon v+(1-2 \varepsilon) N\left(\frac{v}{1-2 \varepsilon}\right) .
\end{aligned}
$$

Let $\varepsilon \rightarrow 0$, we have

$$
u v \leq M(u)+N(v) .
$$


In the following, we will prove sufficiency of the equality.

By the result in Step II, we have

$$
M_{1}(u)+N_{1}\left(p_{1}(u)\right)=u p_{1}(u) .
$$

Therefore,

$$
\begin{aligned}
M(u) & +N(p(u)) \\
\leq & \frac{1}{1-\varepsilon} M_{1}(u)+N(p(u)) \\
& =\left(\frac{1}{1-\varepsilon}-1\right) M_{1}(u)+M_{1}(u)+N_{1}\left(p_{1}(u)\right)+N(p(u))-N_{1}\left(p_{1}(u)\right) \\
& =\left(\frac{1}{1-\varepsilon}-1\right) M_{1}(u)+u p_{1}(u)+N(p(u))-N_{1}\left(p_{1}(u)\right) \quad(\text { by }(19)) \\
& \left.\leq\left(\frac{1}{1-\varepsilon}-1\right) M_{1}(u)+u p_{1}(u)+N(p(u))-\int_{0}^{p_{1}(u)}\left(q\left(\frac{s}{1+2 \varepsilon}\right)-\varepsilon\right) d s \quad \text { (by }(18)\right) \\
& =\left(\frac{1}{1-\varepsilon}-1\right) M_{1}(u)+u p_{1}(u)+\int_{0}^{p(u)} q(s) d s-(1+2 \varepsilon) \int_{0}^{\frac{p_{1}(u)}{1+2 \varepsilon}} q(s) d s+\varepsilon p_{1}(u) \\
& =\left(\frac{1}{1-\varepsilon}-1\right) M_{1}(u)+u p_{1}(u)+N(p(u))-(1+2 \varepsilon) N\left(\frac{p_{1}(u)}{1+2 \varepsilon}\right)+\varepsilon p_{1}(u) .
\end{aligned}
$$

Let $\varepsilon \rightarrow 0$, together with expression (13), we get $p_{1}(u) \rightarrow p(u), M_{1}(u) \rightarrow M(u)$, and $N\left(\frac{p_{1}(u)}{1+2 \varepsilon}\right) \rightarrow N(p(u))$ since $N(v)$ is continuous.

Therefore,

$$
M(u)+N(p(u)) \leq u p(u) .
$$

On the other hand, we have got the inequality $u v \leq M(u)+N(v)$.

Let $v=p(u)$, we have

$$
u p(u) \leq M(u)+N(p(u)) .
$$

Therefore, together with expression (20), we have

$$
M(u)+N(p(u))=u p(u) .
$$

That is, the sufficiency of the equality holds.

\section{Competing interests}

The authors declare that they have no competing interests.

\section{Authors' contributions}

LX conceived of the study, XZ participated in its design and study. All authors read and approved the final manuscript.

\section{Acknowledgements}

This research was partially supported by the National Natural Science Foundation of China (Grant No: 11271245, and Grant No: 11301397), and the Natural Science Foundation Guangdong Province of China (2012KJCX0101). 


\section{References}

1. Pei, LW: Typical Problems and Methods in Mathematical Analysis. Higher Education Press, Beijing (2000) (in Chinese)

2. Wu, CX, Wang, TQ, Chen, ST, Wang, YW: Geometry of Orlicz Spaces. Harbin Institute of Technology Press, Harbin (1986) (in Chinese)

3. Chen, ST: Geometry of Orlicz Spaces. Dissertations Mathematicae Warszawa, Warszawa (1996)

4. Chen, $\mathrm{S}, \mathrm{He}, \mathrm{X}, \mathrm{Hudzik}, \mathrm{H}, \mathrm{Kaminska}$, A: Monotonicity and best approximation in Orlicz-Sobolev spaces with the Luxemburg norm. J. Math. Anal. Appl. 344, 687-698 (2008)

5. Gong, W, Shi, Z: Drop proper ties and approximative compactness in Orlicz-Bochner function spaces. J. Math. Anal. Appl. 344, 748-756 (2008)

6. Shi, Z, Gong, W: Monotone points in Orlicz-Bochner function spaces. Math. Appl. 23(2), 376-383 (2010)

7. Liu, CY, Shi, ZR: U Properties in Orlicz spaces. J. Math. Phys. 31(2), 328-334 (2011) (in Chinese)

8. Shi, ZR, Liu, CY: Noncreasy and uniformly noncreasy Orlicz-Bochner function spaces. Nonlinear Anal. 74, 6153-6161 (2011)

9. Jang, ZJ: Theory of Functions of a Real Variable. Higher Education Press, Beijing (1994) (in Chinese)

doi:10.1186/1029-242X-2013-437

Cite this article as: Xianqiang and Zhiping: A kind of extension of the famous Young inequality. Journal of Inequalities and Applications 2013 2013:437.

\section{Submit your manuscript to a SpringerOpen ${ }^{\circ}$ journal and benefit from:}

- Convenient online submission

- Rigorous peer review

- Immediate publication on acceptance

- Open access: articles freely available online

- High visibility within the field

- Retaining the copyright to your article 